\title{
Avaliação da qualidade de vida e do sofrimento psíquico de pacientes com diferentes dermatoses em um centro de referência em dermatologia no sul do país
}

\author{
Evaluation of the quality of life and psychological distress of patients with \\ different dermatoses in a dermatology referral center in southern Brazil
}

Maria Laura Taborda ${ }^{1}$

Kelly Abreu Machado Teixeira ${ }^{3}$

Elisângela de Quevedo Welter

\author{
Magda Blessmann Weber ${ }^{2}$ \\ Alice Paixão Lisboa ${ }^{4}$
}

Resumo: FundAMENTOS: Algumas dermatoses repercutem, pela sua aparência, na vida pessoal, profissional e social dos pacientes. Faz-se necessária a valorização desse tema visando-se à avaliação global desses indivíduos, para o estabelecimento de uma abordagem terapêutica mais eficaz.

Oвjetivos: Avaliar a qualidade de vida e a frequência de sofrimento psíquico nos pacientes em um centro de Dermatologia.

MÉToDos: Estudo transversal. Após consulta, aplicou-se o Índice de Qualidade de Vida para Dermatologia (DLQI) e o Self-Reported Questionnaire (SRQ-20).

Resultados: Foram entrevistados 1.000 pacientes. Não houve efeito da dermatose sobre a qualidade de vida em 21,5\% deles; 34,2\% apresentaram "pouco efeito", 21,6\%, "efeito moderado", 19,3\%, "grande efeito" e 3,4\%, efeito extremo sobre a qualidade de vida. A correlação entre sofrimento psíquico e dermatoses não mostrou associação estatística significativa. Comparando-se os dois questionários, houve associação significante entre presença de sofrimento psíquico e pior qualidade de vida $(\mathrm{p}<0,001)$. Os pacientes com sofrimento psíquico referiram grande efeito ou efeito extremo sobre a qualidade de vida.

CONCLUSÃO: Este estudo ressalta que várias doenças dermatológicas causam impacto sobre a qualidade de vida e sobre o estado psíquico do indivíduo afetado, influenciando na sua saúde geral, na evolução da dermatose e na resposta às terapêuticas instituídas.

Palavras-chave: Dermatologia; Estresse psicológico; Qualidade de vida

\footnotetext{
Abstract: BACKGROUND: Some dermatoses affect the personal, professional and social lives of patients as a result of their unsightliness. It is important to pay appropriate attention in the overall evaluation of these individuals in order to implement a more effective therapeutic approach.

Овлестілеs: To evaluate the quality of life and the incidence of psychological distress in patients receiving care at a dermatology clinic.

MethoDs: A cross-sectional study. Following consultation, the Dermatology Life Quality Index (DLQI) and the Self Report Questionnaire (SRQ-20) were applied.

RESUlTS: One thousand patients were interviewed, $77.2 \%$ of whom were women. Most patients were in the 41 60 year age-group. Dermatoses had no effect at all on the quality of life of $21.5 \%$ of patients, a small effect in $34.2 \%$ of cases, a moderate effect in $21.6 \%$, a very large effect in $19.3 \%$ and an extremely large effect in $3.4 \%$ of patients. No statistically significant correlation was found between psychological distress and dermatoses. Comparison of the two questionnaires showed a statistically significant association between the presence of psychological distress and poorer quality of life $(\mathrm{p}<0.001)$. Patients with psychological distress reported that their skin condition had a very large or extremely large effect on their quality of life.

CONCLUSION: This study highlights the fact that many skin diseases affect the quality of life and psychological state of the sufferer, affecting his/her health in general, the progression of the skin condition and the patient's response to therapy.

Keywords: Dermatology; Psychological stress; Quality of life

Recebido em 20.8.2009.

Aprovado pelo Conselho Consultivo e aceito para publicação em 17.12.2009.

* Trabalho realizado no Ambulatório do Serviço de Dermatologia da Universidade Federal de Ciências da Saúde de Porto Alegre (UFCSPA)

Conflito de interesse: Nenhum / Conflict of interest: None

Suporte financeiro / Financial funding: : Universidade Federal de Ciências da Saúde de Porto Alegre (UFCSPA)

Mestranda do Programa de Pós-Graduação em Ciências Médicas da Universidade Federal de Ciências da Saúde de Porto Alegre (UFCSPA), dermatologista especialista pela Sociedade Brasileira de Dermatologia - Porto Alegre (RS), Brasil.

Professora adjunta doutora de Dermatologia da Universidade Federal de Ciências da Saúde de Porto Alegre (UFCSPA), doutora em Dermatologia pela Universidade Federal do Rio Grande do Sul (UFRGS) - Porto Alegre (RS), Brasil.

Mestranda do Programa de Pós-Graduação em Patologia da Universidade Federal de Ciências da Saúde de Porto Alegre (UFCSPA), dermatologista especialista pela Sociedade Brasileira de Dermatologia - Porto Alegre (RS), Brasil.

Médica - Porto Alegre (RS), Brasil.

Médica dermatologista especialista pela Sociedade Brasileira de Dermatologia - Porto Alegre (RS), Brasil.
}

(C)2010 by Anais Brasileiros de Dermatologia 


\section{INTRODUÇÃO}

Várias dermatoses geram impacto no estado emocional, nas relações sociais e nas atividades cotidianas dos pacientes, em razão dos estigmas causados pela aparência das lesões. ${ }^{1}$ Estima-se que, pelo menos, um terço dos pacientes com doenças de pele tenha repercussões emocionais à sua dermatose. ${ }^{2}$ Portanto, torna-se relevante a avaliação do sofrimento dos pacientes relacionado às suas dermatoses e, também, a compreensão das alterações de qualidade de vida (QOL). Diversos fatores influenciam o grau de impacto que as doenças terão sobre a QOL do doente: características individuais, história natural da doença, região corporal afetada e tempo de doença prévio ao diagnóstico. ${ }^{3}$

Estudos evidenciam que dermatoses de baixa morbidade provocam impacto semelhante a doenças como asma e epilepsia ${ }^{4}$ demonstrando que doenças de pele prejudicam a autoimagem e têm potencial para conduzir à depressão e à ansiedade tanto quanto doenças sistêmicas graves. Apesar de não serem ameaçadoras à vida nem fisicamente debilitantes, podem afetar gravemente funções psicológicas e sociais dos indivíduos. ${ }^{5}$

Por serem aparentes, as dermatoses repercutem facilmente na vida pessoal, profissional e social de seus portadores. Em vista disso, faz-se necessária a valorização desse tema visando à avaliação global desses indivíduos para o estabelecimento de uma terapêutica mais completa e efetiva. Dessa forma, objetiva-se avaliar a qualidade de vida e a frequência de sofrimento psíquico nos pacientes em um centro de referência em Dermatologia no Sul do país.

\section{MATERIAIS E MÉTODOS}

Estudo transversal com amostra composta por todos os pacientes que consultaram o serviço entre os meses de janeiro e julho de 2007. Foram considerados fatores de exclusão: idade menor de 18 anos e qualquer doença capaz de interferir na cognição. Após consulta dermatológica de rotina, aplicou-se o Índice de Qualidade de Vida para Dermatologia (DLQI), instrumento validado para uso no Brasil. O DLQI consiste em dez itens divididos em seis categorias: sintomas e sentimentos, atividades diárias, lazer, trabalho/escola, relações interpessoais e tratamento. As respostas geram escores entre zero e três e o cálculo final é um somatório simples desses escores, com os índices maiores indicando pior qualidade de vida relacionada à doença.

Nesse momento, os pacientes também responderam o Self-Reported Questionnaire (SRQ-20), instrumento de triagem de doença mental constituído de 20 perguntas que tem sido utilizado em locais de cuidados primários com a saúde. O escore em SRQ-
20, variável contínua, de 0 (zero) a 20 (vinte), foi dicotomizado utilizando-se pontos de corte $7 / 8$ para homens e 8/9 para mulheres. Escores acima dos pontos de corte denotam sofrimento psíquico. Esse instrumento foi elaborado pela Organização Mundial de Saúde (OMS) e está validado para uso no Brasil. As seguintes variáveis foram analisadas (mediante o teste t de Student e o teste qui-quadrado de Pearson): dermatose, idade, sexo do paciente, tempo de evolução da doença e tempo decorrido do diagnóstico inicial, sendo considerada significativa a associação para $\mathrm{p}<$ 0,05. O projeto foi aprovado pelo Comitê de Ética em Pesquisa da Universidade Federal de Ciências da Saúde de Porto Alegre (UFCSPA). Os dados foram armazenados em banco de dados anônimo e analisados com o aplicativo SPSS-12.

\section{RESULTADOS}

Avaliaram-se 1.000 pacientes no período determinado. A amostra foi composta majoritariamente por mulheres $(77,2 \%)$, sendo a faixa etária predominante entre 41 e 60 anos, com média de idade $47,3 \pm 16,5$ anos. O tempo médio de doença foi de 6,2 $\pm 9,7$ anos. As dermatoses mais prevalentes foram micoses superficiais $(13,5 \%)$, tumores cutâneos benignos $(11,9 \%)$, melasma e melanoses adquiridas $(9,3 \%)$, dermatoses pré-malignas $(8,0 \%)$, nevos melanocíticos $(5,9 \%)$, acne $(5,2 \%)$ e tumores cutâneos malignos $(5,0 \%)$.

Quanto ao resultado do DLQI total, a mediana foi de 5,0 (2,0-10,0), mostrando efeito baixo das dermatoses na qualidade de vida. A média do escore geral foi de $6,5 \pm 6,0$, também evidenciando pouco efeito. Não houve efeito da doença dermatológica na QOL em $21,5 \%$ dos doentes (215 pacientes). Nos demais, a dermatose determinou algum efeito, sendo que $34,2 \%$ deles apresentaram "pouco efeito", 21,6\%, "efeito moderado", 19,3\%, "grande efeito" e 3,4\% obtiveram DLQI >20, ou seja, efeito extremo sobre a qualidade de vida.

Analisando-se separadamente os seis domínios do DLQI, apenas o referente a "sintomas e sentimentos" agrupou pacientes com escores mais altos. Os demais domínios associaram-se a escore "nenhum efeito" (Tabela 1).

Relacionando-se o sexo dos pacientes com o escore geral do DLQI, não se encontrou associação estatisticamente significativa; entretanto, observouse uma tendência do sexo feminino a se enquadrar na faixa "pouco efeito" da doença sobre a qualidade de vida.

Comparando-se o sexo com os domínios específicos do DLQI, encontraram-se diferenças estatisticamente significantes nos domínios "tratamento" e 
TABela 1: Escore do DLQI em cada domínio

\begin{tabular}{|c|c|c|c|c|c|c|}
\hline \multirow[b]{2}{*}{ DLQI } & \multicolumn{6}{|c|}{ Qualidade de vida (\%) } \\
\hline & $\begin{array}{l}\text { Sintomas e } \\
\text { sentimentos }\end{array}$ & Atividades diárias & Lazer & Trabalho/escola & $\begin{array}{l}\text { Relações } \\
\text { interpessoais }\end{array}$ & Tratamento \\
\hline 0 & 14,4 & 43,6 & 47,4 & 70,4 & 61,5 & 74,2 \\
\hline 1 & 25,4 & 20,5 & 22,0 & 13,3 & 16,4 & 16,5 \\
\hline 2 & 21,2 & 14,9 & 10,7 & 3,9 & 9,7 & 5,9 \\
\hline 3 & 18,3 & 8,5 & 8,9 & 12,4 & 5,6 & 3,4 \\
\hline 4 & 11,8 & 6,4 & 5,2 & & 3,8 & \\
\hline 5 & 5,4 & 3,7 & 2,5 & & 1,1 & \\
\hline 6 & 3,5 & 2,4 & 3,3 & & 1,9 & \\
\hline Total & 100,0 & 100,0 & 100,0 & 100,0 & 100,0 & 100,0 \\
\hline
\end{tabular}

"trabalho/escola". O tratamento das dermatoses não alterou a QOL nas mulheres $(p=0,036)$ e os homens foram afetados, principalmente, no domínio "trabalho/escola" $(p=0,025)$.

Correlacionando-se o DLQI com as doenças dermatológicas, evidenciou-se que a alopécia, as dermatoviroses e os tumores cutâneos malignos não causam efeito sobre a QOL dos pacientes e que os nevos melanocíticos e os tumores cutâneos benignos apresentam pouco efeito nela. As infecções bacterianas mostraram-se associadas a um "efeito moderado", os eczemas de contato, a psoríase, o prurido e a urticária estavam associados a um "grande efeito" e as colagenoses, a "efeito extremo" na QOL dos indivíduos estudados $(p<0,001)$.

Em relação a cada um dos domínios do DLQI e às alterações causadas pelas diferentes dermatoses, ficou evidenciado que, no domínio "sintomas e sentimentos", houve significância estatística, demonstrando alteração na QOL no eczema de contato, nas micoses superficiais e na psoríase $(\mathrm{p}<0,001)$. No domínio "atividades diárias", essa relação ocorreu com as doenças do colágeno, com o eczema atópico, com o eczema de contato, com o prurido, com a urticária e com a psoríase $(\mathrm{p}<0,001)$. Esta última, bem como o eczema de contato e as doenças do colágeno, interferiram no domínio "lazer" $(p=0,002)$. Infecções bacterianas, doenças do colágeno, eczema de contato e psoríase alteraram o domínio "trabalho e escola" $(\mathrm{p}<0,001)$, enquanto que prurido, urticária, eczema de contato e infecções bacterianas tiveram influência no domínio "relações interpessoais" $(\mathrm{p}<0,001)$.

$\mathrm{Na}$ análise geral do instrumento SRQ em relação ao sexo dos pacientes, não se detectou associação estatística significativa ( $\mathrm{p}>0,05)$; no entanto, a significância mínima obtida pode ser considerada limítrofe $(0,05<$ p $<$ associação tendência $><$ p $<$ associação tendência $><\mathrm{p}<\mathrm{p}>$.
No que diz respeito aos resultados do SRQ segundo a faixa etária, identificou-se associação estatística significativa nas mulheres $(\mathrm{p}<0,05)$. Mulheres entre 18 e 25 anos e entre 26 e 40 anos não apresentaram sofrimento psíquico, enquanto que, nas faixas etárias entre 41 e 60 anos e 61 anos ou mais, encontrou-se relação com sofrimento psíquico (Tabela 2). Na avaliação do sexo masculino, não se detectou associação significativa. Contudo, pela análise dos resíduos ajustados, evidencia-se uma breve tendência de associação da faixa etária de 61 anos ou mais com os valores de SRQ acima do ponto de corte.

Analisando-se o sofrimento psíquico (SRQ) e sua relação com as dermatoses em geral, descobriu-se que, no que se refere ao total da amostra, independentemente do sexo, não houve associação estatística significativa $(p>0,05)$. Contudo, identificaram-se tendências de associação com algumas das doenças, de forma que micoses superficiais e vitiligo se mostraram associadas à ausência de sofrimento, ao passo que acne, eczema de contato e psoríase estavam associados com sofrimento psíquico.

Quanto ao sexo, as mulheres mostraram tendência de associação de sofrimento psíquico com psoríase e eczemas em geral, enquanto que não houve sofrimento quando elas apresentavam acne. Entre os homens, por outro lado, encontrou-se tendência de associação de sofrimento psíquico com eczema atópico, prurido e urticária, mas não com acne, tumores cutâneos malignos, vitiligo e melasma.

Comparando-se os resultados dos dois questionários, encontrou-se associação estatística significativa entre a presença de sofrimento psíquico e pior qualidade de vida $(\mathrm{p}<0,001)$ (Tabela 3$)$. Os pacientes com sofrimento psíquico referiram "grande efeito" ou "efeito extremo" sobre a QOL, enquanto que os pacientes sem sofrimento se enquadraram nas categorias "pouco efeito" ou "sem efeito" do Índice de 
TABela 2: Sofrimento psíquico em mulheres por faixa etária

\begin{tabular}{|c|c|c|c|c|c|c|}
\hline \multirow{3}{*}{ Faixa etária } & & & \multicolumn{4}{|c|}{ SRQ } \\
\hline & \multicolumn{2}{|c|}{ Total } & \multicolumn{2}{|c|}{ Negativo } & \multicolumn{2}{|c|}{ Positivo } \\
\hline & N. & $\%$ & N. & $\%$ & $\mathrm{~N}$. & $\%$ \\
\hline De 18 a 25 anos & 74 & 13,1 & 61 & 16,0 & 13 & 7,2 \\
\hline De 26 a 40 anos & 134 & 23,8 & 92 & 24,1 & 42 & 23,2 \\
\hline De 41 a 60 anos & 236 & 41,9 & 147 & 38,5 & 89 & $49,2^{*}$ \\
\hline 61 anos ou mais & 119 & 21,1 & 82 & 21,5 & 37 & $20,4^{*}$ \\
\hline Total & 368 & 100,0 & 216 & 100,0 & 152 & 100,0 \\
\hline
\end{tabular}

Teste qui-quadrado de Pearson com correção de continuidade. Feminino: $\mathrm{c}^{2}$ calc $^{2}=10,662 ;{ }^{*} \mathrm{p}=0,014$

Qualidade de Vida para Dermatologia. Analisando-se as duas variáveis segundo o sexo, observou-se que tanto os homens quanto as mulheres que apresentavam sofrimento psíquico também referiram pior qualidade de vida. Os homens mostraram associação com as categorias "efeito moderado", "grande efeito" e "efeito extremo" e as mulheres, apenas com as duas últimas citadas.

\section{DISCUSSÃO}

$\mathrm{Na}$ análise do DLQI, verificou-se que $78,5 \%$ dos pacientes relataram algum efeito da doença sobre a qualidade de vida, sendo que $22,7 \%$ demonstraram efeito grande ou extremo. A dermatite de contato foi a dermatose que apresentou a pior qualidade de vida em todos os domínios (sintomas e sentimentos, atividades diárias, lazer, trabalho/escola, relações interpessoais, Tratamento), além de demonstrar tendência de associação com sofrimento psíquico. Esse dado já havia sido descrito por Agner et al., quando demonstraram que a QOL é afetada significativamente em pessoas com eczema de contato nas mãos, independentemente do sexo. ${ }^{6}$ É provável que o caráter recidi- vante e incapacitante dessa dermatose possa ter contribuído para tais achados. Além disso, o tratamento da dermatite de contato, muitas vezes, exige mudanças de hábitos de vida e afastamento do trabalho, prejudicando o orçamento doméstico do paciente e de seus dependentes e interferindo até mesmo na sua qualidade de vida.

A psoríase também demonstrou causar prejuízo na QOL de seus portadores, provavelmente, por se tratar de dermatose crônica e, por vezes, estigmatizante. Não foram encontrados dados na literatura referentes a esse tema.

A associação entre o questionário SRQ e as doenças dermatológicas estudadas não se mostrou estatisticamente significante. Verificou-se, apenas, tendência de associação entre acne, eczema de contato e psoríase com presença de sofrimento psíquico. A acne e a psoríase são dermatoses que ocorrem, em geral, em áreas visíveis e que frequentemente geram sentimentos negativos por serem inestéticas, modificando hábitos rotineiros do paciente, como sair com amigos e ir a festas. Assim, a autoestima do paciente e suas atividades diárias podem ficar prejudicadas signi-

TABELA 3: Qualidade de vida e sofrimento psíquico

\begin{tabular}{|c|c|c|c|c|c|c|}
\hline \multirow{3}{*}{ DLQI } & \multicolumn{4}{|c|}{ SRQ } & & \\
\hline & \multicolumn{2}{|c|}{ Negativo } & \multicolumn{2}{|c|}{ Positivo } & \multicolumn{2}{|l|}{ Total } \\
\hline & $\mathrm{n}$ & $\%$ & $\mathrm{n}$ & $\%$ & $\mathrm{n}$ & $\%$ \\
\hline Sem efeito & 176 & 81,9 & 39 & 18,1 & 215 & 100,0 \\
\hline Pouco efeito & 254 & 74,3 & 88 & 25,7 & 342 & 100,0 \\
\hline Efeito moderado & 141 & 65,3 & 75 & 34,7 & 216 & 100,0 \\
\hline Grande efeito & 103 & 53,4 & 90 & 46,6 & 193 & 100,0 \\
\hline Efeito extremo & 11 & 32,4 & 23 & 67,6 & 34 & 100,0 \\
\hline Total & 685 & 68,5 & 315 & 31,5 & 1.000 & 100,0 \\
\hline
\end{tabular}

$$
c_{\text {calc }}^{2}=65,170{ }^{\circ} \mathbf{p}<0,001
$$


ficativamente. Estudo publicado recentemente por Abdel-Hafez et al. também constatou que a acne vulgar afeta grandemente a QOL e o estado psicológico do indivíduo, apesar de não ser uma dermatose ameaçadora à vida. ${ }^{5}$ Contudo, pode ser prudente considerar o grau de acne para se estabelecer o verdadeiro impacto dessa condição sobre o paciente. ${ }^{4}$ Kaymac et al. verificaram que tratamentos satisfatórios, tópicos ou sistêmicos, diminuíram os sintomas de depressão e ansiedade e melhoraram a qualidade de vida.'

Pelo fato de as micoses superficiais serem doenças facilmente tratadas, faz sentido pensar que não exercem impacto na vida do portador. Entretanto, o vitiligo pode ser avassalador para o paciente, dependendo da extensão e das áreas acometidas, pois, apesar de não ser ameaçador à vida, é doença bastante inestética. É possível que a maior parte dos pacientes do estudo apresentasse a doença em áreas pequenas e cobertas, como acontece, muitas vezes, na área genital, não acarretando prejuízo social e psíquico.

$\mathrm{Na}$ análise entre SRQ e sexo, também não houve associação significativa, mas, após avaliação dos resíduos ajustados, notou-se tendência de associação entre sexo feminino e presença de sofrimento psíquico. Provavelmente, esse achado vai ao encontro do fato de que a prevalência de distúrbios psiquiátricos, como depressão, ocorre mais em mulheres do que em homens. ${ }^{8} \mathrm{O}$ fato de as faixas etárias maiores estarem associadas a maior sofrimento psíquico entre as mulheres é curioso. Pode-se especular que seja devido à influência de outros fatores, como menopausa, diabetes, hipercolesterolemia, hipertensão, que podem aumentar a percepção que a paciente tem de desconforto e de que realmente esteja doente. Muito interessante foi a constatação de que os pacientes com a pior QOL também eram os que tinham maior sofrimento psíquico, independentemente do sexo. Ambos os questionários foram capazes de detectar, de maneira concordante, as pessoas que sentiam maior impacto da dermatose em suas vidas.

\section{CONCLUSÃO}

A partir deste estudo, pode-se perceber a importância de uma avaliação abrangente do paciente para se compreender melhor o quanto e de que formas a doença dermatológica pode prejudicar sua qualidade de vida e sua saúde mental. O impacto psicológico gerado pela dermatose deve ser cuidadosamente avaliado e tratado individualmente, pois, sem dúvida, influenciará a evolução da dermatose e a resposta terapêutica.

\section{REFERÊNCIAS}

1. Finlay AY, Khan GK. Dermatology life quality index (DLQI) - a simple practical measure for routine clinical use. Clin Exp Dermatol. 1994;19:210-6

2. Madhulika A, Aditya K. Psychodermatology: an up date. J Am Acad Dermatol. 1996;34:1030-46.

3. Kadky DL, McCarter K, Achen F, Belsito DV. Quality of life in patients with allergic contact dermatitis. J Am Acad Dermatol. 2003;49:1037-48.

4. Thomas DR. Psychosocial effects of acne. J Cutan Med Surg. 2004;8Suppl4:3-56.

5. Abdel-Hafez K, Mahran AM, Hofny ER, Mohammed KA, Darweesh AM, Aal AA. The impact of acne vulgaris on the quality of life and psychologic status in patients from upper Egypt. Int J Dermatol. 2009;48:280-5.

6. Agner T, Andersen KE, Brandao FM, Bruynzeel DP et al. Hand eczema severity and quality of life: a cross-sectional, multicentre study of hand eczema patients. Contact Dermatitis. 2008;59:43-7.

7. Kaymak Y, Taner E, Taner Y. Comparison of depression, anxiety and life quality in acne vulgaris patients who were treated with either isotretinoin or topical agents. Int J Dematol. 2009:48:41-6.

8. American Psychiatric Association. Diagnostic and statistical manual of mental disorders. 4th ed. Text Revision. Washington, DC: American Psychiatric Association; 2000.

Endereço para correspondência / Mailing Address: Maria Laura Taborda Vila Assunção. Bororó - 55.

91900540 Porto Alegre, RS

Tel: $513395-5716$

Celular: 5199448620

Email:mlaura@taborda.med.br

Como citar este artigo/How to cite this article: Taborda ML, Weber MB, Teixeira KAM, Lisboa AP, Welter EQ. Avaliação da qualidade de vida e do sofrimento psíquico de pacientes com diferentes dermatoses em um centro de referência em dermatologia no sul do país. An Bras Dermatol. 2010;85(1):52-6. 